\title{
Hypercholesterolemia in Shetland Sheepdogs
}

\author{
Kota SATO ${ }^{1)}$, Hideaki $\mathrm{AGOH}^{1)}$, Takahiro KANESHIGE ${ }^{1)}$, Yoshiaki HIKASA ${ }^{1)}$ and Katsumoto KAGOTA ${ }^{1)}$ \\ ${ }^{1)}$ Department of Veterinary Internal Medicine, Faculty of Agriculture, Tottori University, Koyama, Tottori 680-8553, Japan
}

(Received 9 March 2000/Accepted 28 August 2000)

ABSTRACT. Plasma lipoprotein cholesterol in 64 clinically healthy Shetland sheepdogs was evaluated to assess whether the breed is more susceptible to hypercholesterolemia. The incidence of hypercholesterolemia was clearly higher in Shetland sheepdogs and mean plasma cholesterol level was significantly higher in Shetland sheepdogs than in control dogs. Blood biochemical examinations did not evidence the abnormalities, which imply the causative disorders, and thyroid hormone levels were not significantly different from the controls. These results suggest that the cholesterolemia is a primary disorder. Cholesterol fractionation by agarose gel electrophoresis and ultracentrifugation revealed that accumulation of $\alpha_{2}$-migrating lipoproteins was the common characteristic of dogs showing cholesterol level over $250 \mathrm{mg} / \mathrm{d} l$ in the breed. Increase in pre $\beta \sim \beta$-lipoproteins was also found in Shetland sheepdogs with marked hypercholesterolemia over $500 \mathrm{mg} / \mathrm{d} l$. Therefore, Shetland sheepdogs may include more dogs with primary disorders in lipoprotein metabolism, which cause hypercholesterolemia, at least in Japan.

KEY WORDS: cholesterol, high density lipoprotein, low density lipoprotein, Shetland sheepdog.

J. Vet. Med. Sci. 62(12): 1297-1301, 2000

Hypercholesterolemia is characterized by abnormal accumulation of lipoproteins containing cholesterol in plasma. This is closely associated with a pathogeny of atherosclerosis and coronary heart diseases in human [16]. Some primary disorders in cholesterol metabolism such as LDL receptor-deficiency [2] and apolipoprotein disorders [8, 9] have been well documented in human and experimental animals. In dogs, hypercholesterolemia is often found as a secondary consequence following primary disorders such as hypothyroidism, hepatic disorders, and nephrotic syndrome [15]. Primary hyperlipidemia including hypercholesterolemia have been reported in some breeds such as Beagle [14], Briard [17] and Miniature Schnauzer [18] and lesions of atherosclerosis in arterial vessels were found in cholesterol-fed hyperlipidemic dogs when the plasma cholesterol is exceedingly elevated [11]. Although the cause of primary hypercholesterolemia in these dogs have not been well defined except hypothyroidism in Beagle [14], they are thought to be an excellent model to clarifying the molecular basis of hyperlipidemia and athelosclerosis.

Recently, we had found severe hypercholesterolemia $(1,000-2,000 \mathrm{mg} / \mathrm{d} l)$ in several Shetland sheepdogs without obvious clinical manifestations. Since such higher cholesterol level was frequently observed in this breed, we suspected the association of a hereditary factor(s) which is specific for this breed. In the present study, we evaluated the plasma cholesterol level in Shetland sheepdogs which showed no clinical manifestations. In addition, we characterized the lipoprotein-cholesterol in plasma of this breed.

\section{MATERIALS AND METHODS}

Dogs and blood sampling: Clinically healthy Shetland sheepdogs and control dogs (including 21 mongrel dogs, 11 Shiba dogs and 9 Beagle dogs, Table 1), fasted for at least 6 hr, were used. There were no significant differences in both mean age and body weight between Shetland sheepdogs and controls. Venous blood samples were immediately treated with ethylenediaminetetraacetate (EDTA) and plasma were separated by centrifugation at $1,500 \times \mathrm{g}$ for $10 \mathrm{~min}$. When the samples could not be processed immediately, EDTA treated blood was stored at $4^{\circ} \mathrm{C}$ up to $12 \mathrm{hr}$. In this storage condition, blood biochemical tests were not affected. The plasma samples were frozen at $-20^{\circ} \mathrm{C}$ until use. For lipoprotein electrophoresis, plasma samples were used immediately or mixed with sucrose $(25 \% \mathrm{w} / \mathrm{v})$ and frozen at $-20^{\circ} \mathrm{C}$ to prevent degradation of lipoproteins.

Blood chemistry and biochemistry: Plasma total cholesterol and triglyceride were determined by spectrophotometrically using commercial kits (Cholesteroltest E Wako, Triglyceride-test E Wako; Wako Purechem. Industries, Osaka, Japan). Aspartate aminotransferase (AST), alanine aminotransferase (ALT), $\gamma$-glutamyltransferase (GGT), total bilirubin, blood urea nitrogen (BUN) and glucose were determined using Spotchem dry chemistries (Kyoto Daiichi Kagaku, Kyoto, Japan). Plasma total triiodothyronine $\left(\mathrm{T}_{3}\right)$ and thyroxine $\left(\mathrm{T}_{4}\right)$ were determined by double antibody radioimmuno assay kits $\left(\mathrm{T}_{3}\right.$ "Eiken", $\mathrm{T}_{4}$ "Eiken"; Eiken Chem., Tokyo, Japan). When the causative disorders, such as hypothyroidism $\left(\mathrm{T}_{4}<0.5 \mu \mathrm{g} / \mathrm{d} l\right)$, were found in these examinations, the data of the dogs were not used for the comparison of plasma lipid profiles.

Agarose gel electrophoresis of lipoproteins: The plasma samples were separated on agarose gels (TITAN gel-lipo

Table 1. The dogs used in this study

\begin{tabular}{lcc}
\hline & Control & Shetland sheepdogs \\
\hline Number (male, female) & $41(18,23)$ & $64(26,38)$ \\
Age (years, mean \pm S. D.) & $7.5 \pm 2.8$ & $5.8 \pm 3.4$ \\
Body weight $(\mathrm{kg}$, mean \pm S. D.) & $11.0 \pm 2.9$ & $13.3 \pm 4.7$ \\
\hline
\end{tabular}


protein; Helena Laboratories, Beaumont, TX) at $90 \mathrm{~V}$ for 25 min and stained manually with TITAN-S gel cholesterol kit (Helena) according to manufacturer's instruction. The migrating positions $\left(\alpha_{1}, \alpha_{2}\right.$, pre $\beta$ or $\left.\beta\right)$ of the lipoprotein cholesterol in the agarose gels were determined by staining of the plasma proteins with Ponceau $3 \mathrm{R}$ for samples separated in parallel in the same gels.

Fractionation of lipoproteins in plasma by ultracentrifugation: The plasma samples from hypercholesterolemic dogs were separated by sequential ultracentrifugation using RP65T (Hitachi, Tokyo, Japan) at $40,000 \mathrm{rpm}$ for $20 \mathrm{hr}$ at $20^{\circ} \mathrm{C}$. The density ranges of each fraction were $d<1.006$ (very low density lipoprotein; VLDL), $1.006<\mathrm{d}<1.063$ (low density lipoprotein; LDL) and $1.063<\mathrm{d}<1.21$ (high density lipoprotein; HDL) as described [7]. The density was adjusted by addition of $\mathrm{KBr}$. Each lipoprotein fraction was dialyzed against $154 \mathrm{mM}$ $\mathrm{NaCl}, 1 \mathrm{mM}$ EDTA, pH7.4 and analyzed by agarose gel electrophoresis as above.

Statistical analysis: Data were analyzed by Student's $t$-test or Mann-Whitney U test. When the $F$ value was not significant, the Student's $t$-test was used to analyze the difference between each mean value. When a significant $F$ value was found, the Mann-Whitney U test was used for statistical evaluation.

\section{RESULTS}

Figure 1 shows the distribution of the cholesterol levels of Shetland sheepdogs and control dogs. The percentage of Shetland sheepdogs within $>270 \mathrm{mg} / \mathrm{d} l$ (upper limit of reference range, Ref. 11) was $43.8 \%$ which was clearly larger than that of control dogs (12.2\%). Mean plasma cholesterol level was also significantly higher in the breed than in control dogs (Table 2 and Fig. 1). These results demonstrated that the incidence of hypercholesterolemia was clearly higher in Shetland sheepdogs.

The correlation between the cholesterol level and age or sex was not evidenced, indicating that these factors did not affect the hypercholesterolemia in these dogs (data not shown). The blood biochemical examination and

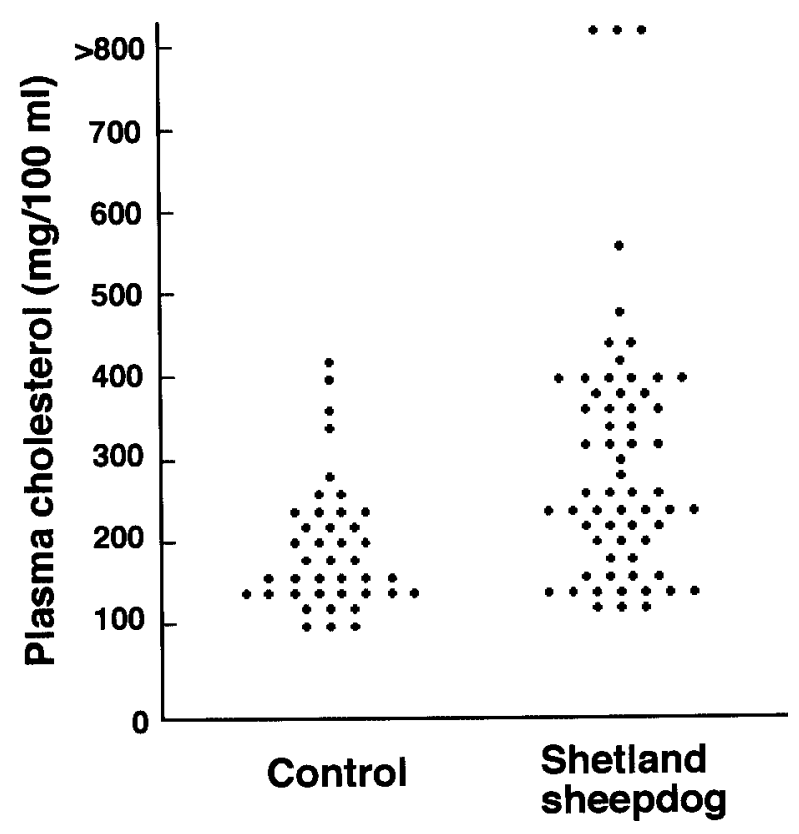

Fig. 1. Distribution of plasma cholesterol level in Shetland sheepdogs. Total plasma cholesterol concentrations were determined in Shetland sheepdogs $(n=62)$ and control dogs $(n=41)$. Cholesterol level of each dog was plotted by every $20 \mathrm{mg} / \mathrm{d} l \mathrm{step}$.

determination of thyroid hormones $\left(\mathrm{T}_{3}\right.$ and $\left.\mathrm{T}_{4}\right)$ did not reveal the abnormality causing cholesterolemia although slight to marked elevation of plasma triglyceride level was observed (Table 2). Correlation between plasma total cholesterol level and triglyceride level was not evidenced (data not shown). Mean levels of thyroid hormones in Shetland sheepdogs were not also significantly different from those of control dogs. In two Shetland sheepdogs, marked hyper-cholesterolemia (over 2,000 $\mathrm{mg} / \mathrm{d} l$ ) and hypertriglycemia (300-400 $\mathrm{mg} / \mathrm{d} l$ ) were found to be caused by hypothyroidism $\left(\mathrm{T}_{4}: 0.1\right.$ and $0.5 \mu \mathrm{g} / \mathrm{d} l, \mathrm{~T}_{3}: 33$ and $46 \mathrm{ng} /$ $\mathrm{d} l$ ). But there was no significant correlation between thyroid hormone levels and plasma cholesterol level in Shetland sheepdogs, indicating that subclinical

Table 2. Blood biochemistry and thyroid hormones in Shetland sheepdogs

\begin{tabular}{llc}
\hline & Control $(\mathrm{n}=41)$ & Shetland sheepdogs $(\mathrm{n}=62)$ \\
\hline Cholesterol $(\mathrm{mg} / \mathrm{d} l)$ & $191 \pm 79$ & $333 \pm 311^{*}$ \\
Triglyceride $(\mathrm{mg} / \mathrm{d} l)$ & $38 \pm 14$ & $104 \pm 86^{*}$ \\
Glucose $(\mathrm{mg} / \mathrm{d} l)$ & $118 \pm 25$ & $111 \pm 46$ \\
BUN $(\mathrm{mg} / \mathrm{d})$ & $19 \pm 9$ & $20 \pm 12$ \\
Total bilirubin $(\mathrm{mg} / \mathrm{d} l)$ & $0.4 \pm 0.3$ & $0.5 \pm 0.6$ \\
ALT $(\mathrm{IU} / l)$ & $48 \pm 21$ & $52 \pm 17$ \\
AST $(\mathrm{IU} / l)$ & $15 \pm 13$ & $12 \pm 10$ \\
GGT $(\mathrm{IU} / l)$ & $25 \pm 12$ & $28 \pm 16$ \\
Plasma total protein $(\mathrm{g} / \mathrm{d} l)$ & $6.8 \pm 0.8$ & $7.2 \pm 0.9$ \\
Triiodothyronine $\left(\mathrm{T}_{3} ; \mathrm{ng} / \mathrm{d} l\right)$ & $73.0 \pm 15.0(\mathrm{n}=10)$ & $87.6 \pm 50.7$ \\
Thyroxine $\left(\mathrm{T}_{4} ; \mu \mathrm{g} / \mathrm{d} l\right)$ & $2.68 \pm 1.40(\mathrm{n}=10)$ & $1.79 \pm 0.80$ \\
\hline
\end{tabular}

Abbreviations: BUN; blood urea nitrogen, ALT; alanine aminotransferase, AST; aspartate aminotransferase, GGT; $\gamma$-glutamyltransferase. $* \mathrm{P}<0.01(t$-test $)$ 


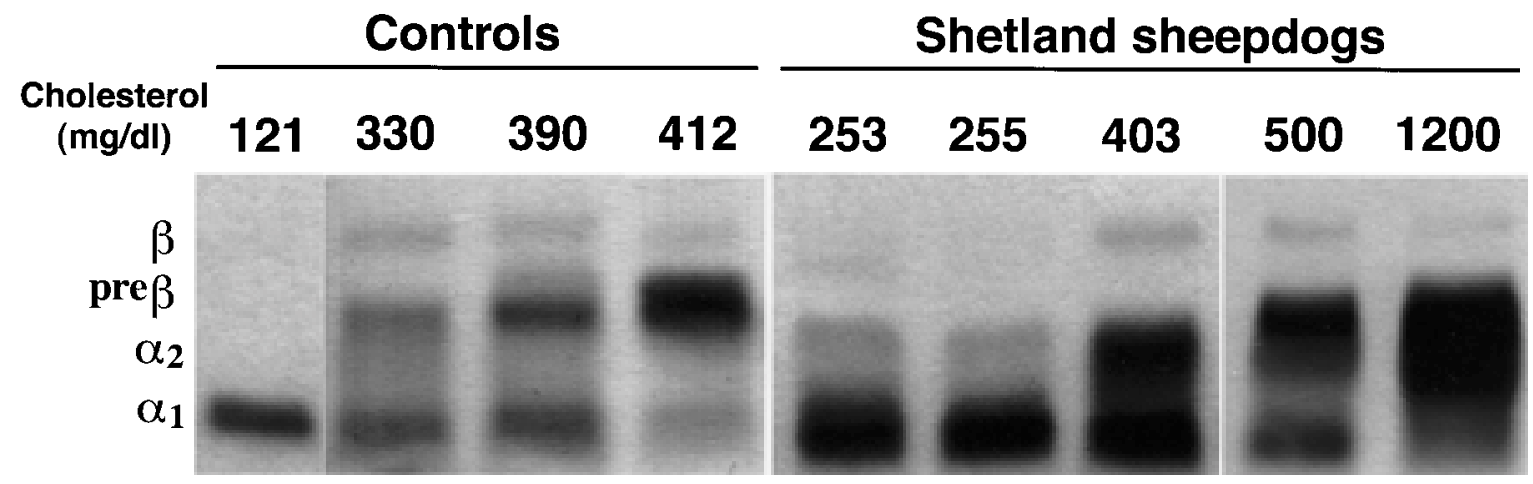

Fig. 2. Representative electrophoretic patterns of plasma cholesterol in Shetland sheepdogs. Aliquots (2 $\mu l$ or $1 \mu l$ for a dog 1,200) of plasma were separated on agarose gels and visualized by cholesterol staining. Number above each lane represents plasma cholesterol concentration $(\mathrm{mg} / \mathrm{d} l)$. Representative patterns of hypercholesterolemic Shetland sheepdogs and control dogs were shown.

hypothyroidism was not a primary disorder in the breed.

The electrophoretic patterns of plasma lipoprotein cholesterol on agarose gels were visualized by cholesterol staining (Fig. 2). The common characteristic in the Shetland sheepdogs was an increase in $\alpha_{2}$-migrating lipoproteins, which was observed in 25 out of 29 Shetland sheepdogs showing cholesterol level over $250 \mathrm{mg} / \mathrm{d} l$. Moreover, increase in pre $\beta \sim \beta$-migrating lipoproteins was observed beside the $\alpha_{2}$-lipoprotein in markedly hypercholesterolemic dogs (over $500 \mathrm{mg} / \mathrm{d} l$ ). In control dogs with normal cholesterol concentration, only an $\alpha_{1}$-migrating band was visualized. The slight to marked rise in pre $\beta \sim \beta$-migrating lipoproteins was observed in four hypercholesteloremic dogs $(330-412 \mathrm{mg} / \mathrm{d} l)$ in the control group.

Figure 3 shows the electrophoretic patterns of lipoproteins fractionated by ultracentrifugation from markedly hypercholesterolemic dogs. The result revealed that both $\alpha_{2}$ - and pre $\beta \sim \beta$ migrating lipoproteins were collected in $1.006<\mathrm{d}<$ 1.063 fraction, which is corresponding to the LDL fraction. Lipoproteins collected in $\mathrm{d}<1.006$ fraction, which is corresponding to VLDL, were migrated near the $\beta$-region in the agarose gels but its band intensity was much lower than $1.006<\mathrm{d}<1.063$ fraction. The $\alpha_{1}$-migrating lipoproteins, which were also observed in control dogs, was collected in $1.063<\mathrm{d}<1.21$ fraction corresponding to HDL.

\section{DISCUSSION}

In the present study, we demonstrated higher incidence of hypercholesterolemia in clinically healthy Shetland sheepdogs. Elevation of plasma cholesterol is often detected in dogs with hypothyroidism, hepatic disorders and nephrotic syndrome [15]. We could not determine the cause of vast majority of hypercholesterolemia in these Shetland sheepdogs since only two dogs could be diagnosed as hypothyroidism by measurement of thyroid hormones. From this observation, we postulated that subclinical disorder in lipoprotein metabolism was causative for hypercholesterolemia in the breed. However, since not all Shetland sheepdogs we examined were cholesterolemic, the

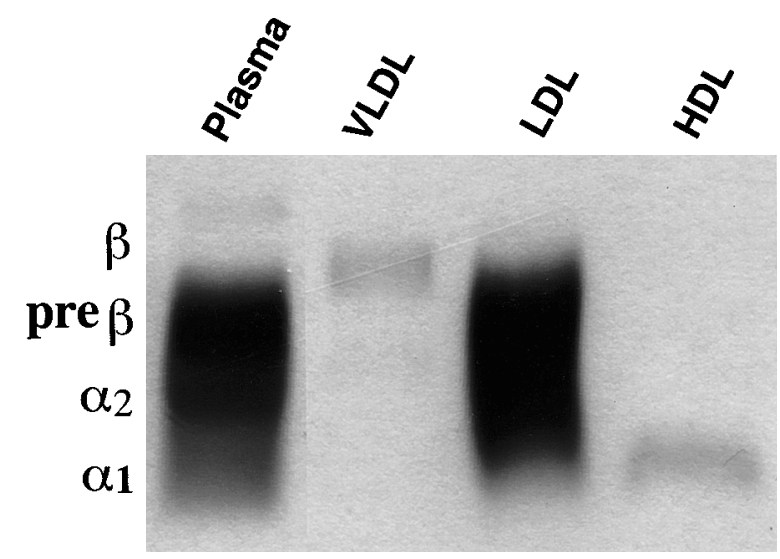

Fig. 3. Representative electrophoretic patterns of ultracentrifugal lipoprotein fractions in the hypercholesterolemic Shetland sheepdog. Plasma of a Shetland sheepdog (total plasma cholesterol was $\approx 1,500 \mathrm{mg} / \mathrm{d} l$ ) was fractionated into very low density lipoprotein (VLDL; d<1.006), low density lipoproteins (LDL; $1.006<\mathrm{d}<1.063$ ) and high density lipoprotein (HDL; $1.063<\mathrm{d}<1.21$ ) by sequential ultracentrifugation. After dialysis against $154 \mathrm{mM} \mathrm{NaCl}, 1 \mathrm{mM}$ EDTA, pH7.4, aliquots were separated on an agarose gel and visualized by cholesterol staining.

abnormality in cholesterol metabolism may be a property of a certain pedigree of the breed found in Japan. Canine primary hypercholesterolemia has been reported in Miniature Schnauzer [18] and in Briards in the United Kingdom [17]. Marked hypertriglycemia is evident in Miniature Schnauzer [18], suggesting that different disorders are suspected in this breed although a few Shetland sheepdogs exhibited similar hypertriglycemia. On the other hand, hypercholesterolemic Briards exhibited elevation of $\alpha_{2}$-migrating $\mathrm{HDL}_{1}$, presumably $\mathrm{HDL}_{\mathrm{C}}$ [17]. The $\mathrm{HDL}_{\mathrm{C}}$ is thought to be a cholesterol-rich $\mathrm{HDL}_{1}$, which is one of the HDL subclass [4], and is characterized by its ultracentrifugal density $(1.025<\mathrm{d}$ $<1.10)$, overlapping LDL $(1.006<\mathrm{d}<1.063)$, and the $\alpha_{2^{-}}$ mobility in electrophoresis [12]. Our lipoprotein analyses revealed that accumulation of $\alpha_{2}$-lipoproteins was more 
commonly observed in Shetland sheepdogs even in slight or marked hypercholesterolemia (Fig. 2). In addition, since this lipoprotein was fractionated in $1.006<d<1.063$ with pre $\beta \sim \beta$ migrating lipoproteins (LDL) by ultracentrifugation (Fig. 3), we propose that these are $\mathrm{HDL}_{\mathrm{C}}$, which is often observed in cholesterol-fed hypercholesterolemic dogs [11]. Such plasma lipoprotein composition was quite similar to Briards [17], suggesting that a certain similar defect was suspected in both breeds. In Briards, hereditary disorder in lipoprotein metabolism was suspected although the precise mechanism was not defined. We also could not clarify causative disorders in lipoprotein metabolism of Shetland sheepdogs at present but some common hereditary disorders may exist in these breeds. The HDL $\mathrm{C}_{\mathrm{C}}$ is thought to be $\mathrm{HDL}_{1}$ which acquire rich cholesterol esters and apolipoprotein $\mathrm{E}$ [5]. Since apolipoprotein E is an important ligand for lipoprotein receptors, such as LDL receptor [6], LDL receptor related protein (LRP) [1], and cell surface proteoglycans [3], a certain pathway for clearance of apoE-rich lipoproteins may be disturbed in Shetland sheepdogs.

In addition, marked hypercholesterolemia (over $500 \mathrm{mg} /$ $\mathrm{d} l$ ) in Shetland sheepdogs was characterized by increase in pre $\beta \sim \beta$-lipoproteins (Fig. 2), which was similar to hypercholesterolemia found in control dogs we examined in this study (Fig. 2). While the cholesterol level of VLDL ( $\mathrm{d}<$ 1.006) fraction was very low, LDL fractions $(1.006<\mathrm{d}<$ $1.063)$ contained vast majority of lipoprotein-cholesterol observed as pre $\beta \sim \beta$ lipoproteins and $\alpha_{2}$-lipoproteins in agarose gels (Fig. 3). This suggests that not only $\alpha_{2}$-lipoproteins (presumably $\mathrm{HDL}_{\mathrm{C}}$ ) but also LDL were accumulated in dogs with marked hypercholesterolemia. Therefore, various disorders may be related to the abnormality in cholesterol metabolism in this breed.

Increase in plasma cholesterol is known to be atherogenic in mammals [16]. In mild hypercholesterolemic Shetland sheepdogs, only increase in $\alpha_{2}$-lipoproteins, presumably HDL $_{\mathrm{C}}$, was observed, while pre $\beta \sim \beta$-migrating LDL lipoproteins were also elevated in severe hypercholesterolemic dogs of the breed. Since the elevation of $\mathrm{HDL}_{\mathrm{C}}$ cholesterol induces accumulation of cholesterol in smooth muscle cells as well as LDL [11], the elevation of $\mathrm{HDL}_{\mathrm{C}}$ level may be a risk factor of cardiovascular diseases in these dogs. In cholesterol-fed dogs, raised cholesterol level in LDL and $\mathrm{HDL}_{\mathrm{C}}$ fractions was accompanied with atherosclelosis in arterial vessels when the plasma cholesterol level reached up to $750 \mathrm{mg} / \mathrm{d} l$ [13]. Since plasma cholesterol level over 750 $\mathrm{mg} / \mathrm{d} l$ was also observed in several Shetland sheepdogs we examined, increase in plasma cholesterol could be atherogenic in these dogs. In our clinical case, a Shetland sheepdog with hypercholesterolemia $(\approx 1,500 \mathrm{mg} / \mathrm{d} l)$ possessed some lesions of atherosclerosis in arterial walls of several tissues although dogs are generally resistant to atherosclerosis (unpublished observation). In addition, it should be noted that exceedingly higher plasma cholesterol level (over 2,000 $\mathrm{mg} / \mathrm{d} l$ ) was found in the dogs with hypothyroidism, suggesting that primary disorders in lipoprotein metabolism may additively elevate the plasma cholesterol in these dogs.
Thus, a certain hereditary factor present in some Shetland sheepdogs might also accelerate accumulation of cholesterol in secondary hypercholesterolemia. These observations indicate that dogs with hypercholesterolemia in this breed may be more susceptible to atherosclerosis.

In conclusion, higher incidence of hypercholesterolemia was found in Shetland sheepdogs. In addition, the accumulation of cholesterol-rich HDL is likely to be more common in the hypercholesterolemia found in Shetland sheepdogs from slight to marked degrees. Therefore, Shetland sheepdogs may include more dogs with primary disorders in lipoprotein metabolism, which cause hypercholesterolemia, at least in Japan.

ACKNOWLEDGMENTS. We would like to appreciate Drs. A. Haruna, Y. Sumisaki (Haruna animal Hospital, Tsuyama), N. Yamane, (Yamane Animal Hospital, Tottori) and H. Eguchi (Eguchi Animal Hospital, Tottori) for their cooperation in sample collection. This work was supported by the grant from ONO Medical Research Foundation.

\section{REFERENCES}

1. Beisiegel, U., Weber, W., Ihrke, G., Herz, J. and Stanley, K. K. 1989. The LDL-receptor-related protein, LRP, is an apolipoprotein E-binding protein. Nature (Lond.) 341: 162-164.

2. Brown, M. S. and Goldstein, J. L. 1986. A receptor-mediated pathway for cholesterol homeostasis. Science 232: 34-47.

3. Fan, J., Ji, Z. S., Huang, Y., de Silva, H., Sanan, D., Mahley, R. W., Innerarity, T. L. and Taylor, J. M. 1998. Increased expression of apolipoprotein $\mathrm{E}$ in transgenic rabbits results in reduced levels of very low density lipoproteins and an accumulation of low density lipoproteins in plasma. J. Clin. Invest. 101: 2151-2164.

4. Fong, B. S., Despres, J. P., Julien, P. and Angel, A. 1988. Interactions of high density lipoprotein subclasses $\left(\mathrm{HDL}_{2}\right.$ and $\left.\mathrm{HDL}_{\mathrm{C}}\right)$ with dog adipocytes: selective effects of cholesterol and saturated fat feeding. J. Lipid Res. 29: 553-561.

5. Gordon, V., Innerarity, T. L. and Mahley, R. W. 1983. Formation of cholesterol- and apoprotein E-enriched high density lipoproteins in vitro. J. Biol. Chem. 258: 6202-6212.

6. Havel, R. J. 1995. Chylomicron remnants: hepatic receptors and metabolism. Curr. Opin. Lipidol. 6: 312-316.

7. Havel, R. J., Eder, J. and Bragdon, J. H. 1955. The distribution and chemical composition of ultracentrifugally separated lipoproteins in human serum. J. Clin. Invest. 34: 1345-1353.

8. Humphries, S. E. and Talmud, P. J. 1995. Hyperlipidaemia associated with genetic variation in the apolipoprotein B gene. Curr. Opin. Lipidol. 6: 215-222.

9. Innerarity, T. L., Weisgraber, K. H., Arnold, K. S., Mahley, R. W., Krauss, R. M., Vega, G. L. and Grundy, S. M. 1987. Familial defective apolipoprotein B-100: low density lipoproteins with abnormal receptor binding. Proc. Natl. Acad. Sci. U. S. A. 84: 6919-6923.

10. Kaneko, J. J. 1989. pp. 892-897. Clinical Biochemistry of Domestic Animals, 4th ed., Academic Press, London.

11. Mahley, R. W., Innerarity, T. L., Weisgraber, K. H. and Fry, D. L. 1977. Canine hyperlipoproteinemia and atherosclerosis. Accumulation of lipid by aortic medial cells in vivo and in vitro. Am. J. Pathol. 87: 205-225.

12. Mahley, R. W. and Weisgraber, K. H. 1974. Canine lipoproteins 
and atherosclerosis. I. Isolation and characterization of plasma lipoproteins from control dogs. Circ. Res. 35: 713-721.

13. Mahley, R. W., Weisgraber, K. H. and Innerarity, T. 1974. Canine lipoproteins and atherosclerosis. II. Characterization of the plasma lipoproteins associated with atherogenic and nonatherogenic hyperlipidemia. Circ. Res. 35: 722-733.

14. Manning, P. J. 1979. Thyroid gland and arterial lesions of Beagles with familial hypothyroidism and hyperlipoproteinemia. Am. J. Vet. Res. 40: 820-828.

15. Rogers, W. A. 1977. Lipemia in the dog. Vet. Clin.North. Am. 7 : 637-647.
16. Ross, R. 1993. The pathogenesis of atherosclerosis: a perspective for the 1990s. Nature (Lond.) 362: 801-809.

17. Watson, P., Simpson, K. W. and Bedford, P. G. 1993. Hypercholesterolaemia in briards in the United Kingdom. Res. Vet. Sci. 54: 80-85.

18. Whitney, M. S., Boon, G. D., Rebar, A. H., Story, J. A. and Bottoms, G. D. 1993. Ultracentrifugal and electrophoretic characteristics of the plasma lipoproteins of miniature schnauzer dogs with idiopathic hyperlipoproteinemia. J. Vet. Intern. Med. 7: 253-260. 